\title{
Decentralized Shaping for Pilot Generation and Detection in Opportunistic Communications
}

\author{
Jordi Borràs and Gregori Vazquez \\ Department of Signal Theory and Communications, Technical University of Catalonia (UPC) \\ Building D5, Campus Nord UPC, Jordi Girona 1-3, 08034 Barcelona, Spain \\ Email: \{jordi.borras.pino, gregori.vazquez\}@upc.edu
}

\begin{abstract}
The uncoordinated design of pulse shaping filters for opportunistic communications is addressed. We show that under degrees-of-freedom sensing uncertainties the waveform design problem can be cast as a minimum-norm optimization, admitting hence a closed-form expression. Because designed waveforms are adapted to scenario working conditions, proposed design scheme may be considered in pilot reference signals design to achieve orthogonality, regardless the traditionally considered pilot symbols orthogonality. Hence, the effect of interferences such as pilot contamination is diminished. However, a crucial aspect relies on their detectability. Since each node uses only local observations from the wireless network, the sensed degrees-of-freedom may slightly differ from one node to others. In this paper we prove that, thanks to the existence of some invariances, designed waveforms can be detected by neighboring nodes. Even though degrees-offreedom sensing uncertainties may incur in a performance loss, we propose a least-squares constrained basis pursuit algorithm to reduce the effect of uncertainties by considering only the degreesof-freedom subspace intersection.
\end{abstract}

Index Terms-Opportunistic communications, distributed networks, pulse generation, multi-signal detection, sparse detection.

\section{INTRODUCTION}

Opportunistic communication [1] allows efficient information transmission in distributed networks due to reduced signaling overhead avoiding centralized backbone infrastructures.

Although lots of works have been devoted to analyzing feasible transmission schemes (see, e.g., [2-4]), performance aspects should be also considered. As in any robust communication system, time-references are required to achieve node synchronization or acquire channel state information (CSI) in order to guarantee the desired performance in terms of achievable throughput and secrecy [5-7], and detection and decoding [8].

Classically, time-references are based on offline-designed pilot signals, which do not take network conditions into account. Therefore, the system performance is degraded in very high user-density scenarios dominated by the level of pilot contamination [9]. Even though pilot contamination has been classically studied in the well-known scenario of massive multiple-input multiple-output (MIMO) communications, it is also present in congested distributed networks [6].

Several signal processing techniques have been recently developed to cope with pilot contamination. These techniques

This research has been supported by the Spanish Ministry of Science, Innovation and Universities through project WINTER: TEC2016-76409-C21-R (AEI/FEDER, UE) and fellowship FPI BES-2017-080071, and by the Catalan Government (AGAUR) under grant 2017 SGR 578. are encompassed in the concept of pilot decontamination. In that respect, some works are devoted to reducing (or, ideally, eliminating) the contamination [10], [11]. On the other side, we may find some works that address how pilot contamination can be avoided [12].

In contrast to the aforementioned works where pilot contamination is assumed and the performance is improved by means of pre- and/or post-processing techniques, we present in this paper a scenario-adapted pilot waveform design scheme. Each user of a distributed network acquires a set of observations from the wireless environment and adapts the waveform in such a way that only available resources are used. It is worth noting that the design scheme presented in this paper is also valid in the context of adaptive waveform pulse shaping design.

Once each user has designed its own pilot, the major challenge is how this node can discover neighboring ones without cooperation, using local pilots as time-references. Hence, this paper also deals with the pilot detectability. We show that, under the existence of some invariances, pilots can be detected. The latter is a necessary condition for using locally-designed pilots as time-references in noncooperative distributed systems.

However, the uncoordinated nature of this system incurs in a performance loss due to node-to-node uncertainty. We finally address how receiver can diminish the effects of uncertainties via a sparsity-based subspace dimension reduction. This contribution enables joint multi-waveform detection, justifying the feasibility of proposed pilot scheme in multiuser scenarios.

The rest of this paper is organized as follows. The problem addressed in this paper is formulated in Sec. II. The distributed pulse shaping generation design is tackled in Sec. III. In Sec. IV we formulate the problem of signal detectability and the required invariances to use distributed pilots as reference signals are analyzed in Sec. V. The (multi-) waveform detection and subspace dimension reduction is analyzed in Sec. VI. Numerical results are provided in Sec. VII and the paper is concluded in Sec. VIII.

\section{Problem Statement}

Robust communication systems use time-references as training and polling schemes, to achieve synchronization, identify the channel or assist multiuser detection. Typically, these reference signals are based on offline-designed pilots, which are not adapted to scenario working conditions. In congested networks, using offline-designed pilots is a source of interferences, such as pilot contamination, yielding a poor performance. 


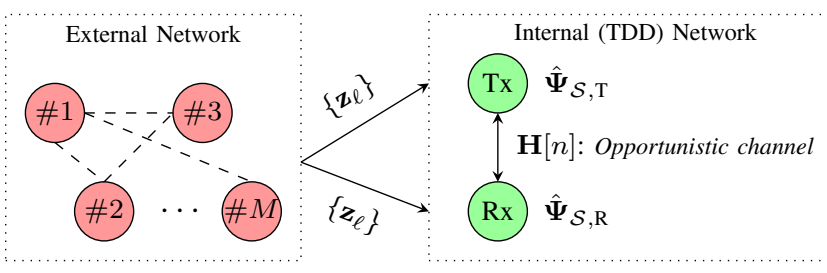

Fig. 1: Context-aware opportunistic communication in a distributed network.

In this work, we address the distributed design of reference signals taking into account the working conditions of a distributed opportunistic communication scenario. Let us consider the scenario depicted in Fig. 1. As we can appreciate, it is composed of two networks. On the one hand, external network is an $M$-user heterogeneous network, where these $M$ users may present different communication formats. Nonetheless, they are simultaneously transmitting information exploiting $D$ degrees-of-freedom (DoF) (cf. [13], [14]). On the other hand, users from internal network wish to setup a point-to-point link without interfering the external-network users. In other words, they want to exploit the unused DoF. To do so, each internalnetwork user acquires $L$ observations of length $N$ from the external network

$$
\mathbf{z}_{\ell}=\mathbf{s}_{\ell}+\boldsymbol{v}_{\ell}, \text { for } \ell=1, \ldots, L,
$$

where $\mathbf{s}_{\ell}$ is the signal transmitted by existing users, and $\boldsymbol{v}_{\ell}$ is the noise distributed as $\boldsymbol{v}_{\ell} \sim \mathcal{C N}\left(\mathbf{0}, \sigma^{2} \mathbf{I}_{N}\right)$.

Taking into account only local observations, each internalnetwork user has to design a pulse shaping filter $\varphi_{i}(n)$, where $i$ indexes the internal user. Let $x(n)$ be the signal received by an arbitrary internal user. It can be written as

$$
x(n)=\sum_{m} \sqrt{S_{\mathrm{R}}} a[m] \varphi_{i}(n-m T)+v(n),
$$

where $a[m]$ is a symbol from a given constellation, $T$ denotes the symbol period and $S_{R}$, the received power. Here, the complex noise $v(n)$ is assumed to be a complex Gaussian random variable, i.e. $v(n) \sim \mathcal{C N}\left(0, \sigma_{v}^{2}\right)$. Due to the generality of (2), we can distinguish two different cases:

(i) When the symbol $a[m]$ is an information symbol, the design of the pulse $\varphi_{i}(n)$ can be seen as an adaptive waveform design problem.

(ii) If $a[m]$ is a known pseudorandom sequence, the problem turns to a distributed pilot waveform design. In that case, in order to avoid spectral lines, this sequence ought to spread the pilot signal energy spectral density.

Once each internal user has designed its pilot $\varphi_{i}(n)$, it first needs to detect the presence of neighboring nodes. Recall the scenario depicted in Fig. 1. Let $\varphi_{\mathrm{T}}(n)$ and $\varphi_{\mathrm{R}}(n)$ be the pulses designed at internal transmitter and receiver, respectively. Thus, the goal of internal system can be cast as

$$
\max _{j}\left|\sum_{n} \varphi_{\mathrm{T}}^{*}(n) \varphi_{\mathrm{R}}(n, j)\right| .
$$

In other words, receiver has to select a pulse $\varphi_{\mathrm{R}}(n)$ from a set and adapt it as a matched filter response to detect the transmitter's signal without transmitter-receiver cooperation, while diminishing the pulse-shaping mismatch due to different knowledge of external-network state at each internal node.

\section{Uncoordinated PUlse Shaping Design}

Hereunder we detail the decentralized pulse shaping design scheme. It is worth noting that it consists in a generalization of particular examples presented by the authors in [15], [16].

Since external-network users employ $D$ DoF, their transmissions belong to a $D$-dimensional subspace. Actually, this subspace corresponds to the occupied DoF (ODoF) subspace. In some frameworks, the latter is known as signal subspace. Henceforth, for simplicity of notation, we refer the ODoF subspace as signal subspace, namely $\mathcal{S}^{D}$. Hence,

$$
\mathbf{s}_{\ell} \in \mathcal{S}^{D}, \forall \ell \in\{1, \ldots, L\}
$$

Whenever $D<N$, there exists a $K$-dimensional subspace, with $K=N-D$, orthogonal to $\mathcal{S}^{D}$. This subspace contains the available DoF (ADoF) for opportunistic communication. Similarly to the ODoF case, ADoF subspace is also known as noise subspace. For simplicity, we will denote ADoF subspace as noise subspace, namely $\mathcal{N}^{K}$.

Taking the latter into account and letting $\Psi_{\mathcal{S}}$ and $\varphi$ be a signal-subspace basis and the opportunistic waveform, the design problem is cast as

$$
\boldsymbol{\Psi}_{\mathcal{S}}^{H} \boldsymbol{\varphi}(\mathbf{r})=\mathbf{0}
$$

where $\mathbf{r}$ is the position vector. In this Section, for simplicity of discussion, we address the pilot design at an arbitrary geographical position $\mathbf{r}$. In the sequel, we drop the dependence on $\mathbf{r}$ for simplicity of notation.

Due to sensing uncertainties, shadowing or channel fading, internal users are not able to obtain an accurate signal-subspace basis. Thus, we may find two possible scenarios:

(i) Internal users overestimate signal-subspace dimension: Internal users will lose transmission opportunities, which is not critical in view of (5).

(ii) Internal users underestimate signal-subspace dimension: This is the critical scenario. Some of the occupied DoF are erroneously sensed as available. Hence, internal users will also use them, leading to a noise enhancement.

Bearing in mind the worst case, a signal-subspace basis can be written as

$$
\boldsymbol{\Psi}_{\mathcal{S}}=\left[\begin{array}{l:l}
\hat{\mathbf{\Psi}}_{\mathcal{S}} & \boldsymbol{\Xi}
\end{array}\right]
$$

where $\hat{\Psi}_{\mathcal{S}}$ stands for the sensed signal-subspace basis, and $\boldsymbol{\Xi}$ represents those DoF that are erroneously sensed as available. In (6), an actual signal-subspace basis can be seen as a columnstacking of orthonormal bases $\hat{\boldsymbol{\Psi}}_{\mathcal{S}}$ and $\boldsymbol{\Xi}$.

It is worth noting that, in view of (6), the signal subspace $\mathcal{S}^{D}$ admits the following decomposition

$$
\mathcal{S}^{D}=\hat{\mathcal{S}}^{\tilde{D}} \cup \mathcal{E}^{\delta},
$$

with $D=\tilde{D}+\delta$. In (7), $\mathcal{E}^{\delta}$ refers to the portion of the actual signal subspace spanned by $\boldsymbol{\Xi}$. Since an arbitrary internal user will only be able to sense $\hat{\Psi}_{\mathcal{S}}$, it will also exploit those DoF belonging to $\mathcal{E}^{\delta}$. Therefore, taking (6) into consideration, the cost function becomes

$$
\min _{\varphi} \max _{\Xi}\left\|\left[\hat{\mathbf{\Psi}}_{\mathcal{S}}: \boldsymbol{\Xi}\right]^{H} \varphi\right\|^{2} .
$$


In order to constrain the uncertainty in our problem, we upperbound the rank of $\Xi$. Furthermore, because (8) admits a trivial solution, we consider the linear predictor condition [17] as a non-trivial design constraint. Thus, (8) becomes

$$
\min _{\boldsymbol{\varphi}} \max _{\boldsymbol{\Xi}}\left\|\left[\hat{\boldsymbol{\Psi}}_{\mathcal{S}} \boldsymbol{\Xi}\right]^{H} \boldsymbol{\varphi}\right\|^{2} \text { s.t. }\|\boldsymbol{\Xi}\|_{\mathrm{F}}^{2} \leq \epsilon \text { and } \mathbf{e}_{n}^{H} \boldsymbol{\varphi}=1
$$

with $\mathbf{e}_{n} \triangleq\left[\begin{array}{lll}\mathbf{0}_{n-1}^{T} & 1 & \mathbf{0}_{N-n}^{T}\end{array}\right]^{T}$. Because $\hat{\mathbf{\Psi}}_{\mathcal{S}}^{H} \boldsymbol{\varphi}=\mathbf{0}$ and defining the orthogonal projector onto $\mathcal{E}^{\delta}$ as $\boldsymbol{\Sigma} \triangleq \boldsymbol{\Xi} \boldsymbol{\Xi}^{H}$, the pulse shaping filter design problem is finally given by

$$
\tilde{\boldsymbol{\varphi}}_{n}=\underset{\boldsymbol{\varphi}}{\arg \min } \max _{\Xi}\|\boldsymbol{\Sigma} \boldsymbol{\varphi}\|^{2} \text { s.t. } \mathbf{e}_{n}^{H} \boldsymbol{\varphi}=1,\|\boldsymbol{\Sigma}\|_{\mathrm{F}}^{2} \leq \epsilon .
$$

Due to lack of space, the derivation of (10) is only sketched herein. The reader is referred to [15], [16] for further details.

By solving the maximization problem in (10), we get

$$
\Sigma_{\text {worst-case }}=\frac{\epsilon}{\|\varphi\|^{2}} \varphi \varphi^{H}
$$

Notice that, in view of (11), $\epsilon=1$, which means that internal users inject all transmitted power into DoF occupied by external users. Then, since $\hat{\Psi}_{\mathcal{S}}^{H} \boldsymbol{\varphi}=\mathbf{0}$, the designed waveforms can be written as a linear combination of the elements of the sensed noise-subspace basis, i.e. $\varphi=\hat{\Psi}_{\mathcal{N}} \boldsymbol{\lambda}$. Hence, (10) yields

$$
\tilde{\boldsymbol{\varphi}}_{n}=\left(\mathbf{e}_{n}^{H} \hat{\boldsymbol{\Psi}}_{\mathcal{N}} \hat{\mathbf{\Psi}}_{\mathcal{N}}^{H} \mathbf{e}_{n}\right)^{-1 / 2} \hat{\boldsymbol{\Psi}}_{\mathcal{N}} \hat{\mathbf{\Psi}}_{\mathcal{N}}^{H} \mathbf{e}_{n}
$$

with $\hat{\mathbf{P}}_{\mathcal{N}}=\hat{\mathbf{\Psi}}_{\mathcal{N}} \hat{\mathbf{\Psi}}_{\mathcal{N}}^{H}$ being the orthogonal projector onto the noise subspace. Notice that the solution in (12) is a normalized column of $\hat{\mathbf{P}}_{\mathcal{N}}$. Since all they are orthogonal to the sensed signal-subspace basis, any of them are a possible solution.

It is worth noting that the proposed design scheme reduces to a classical minimum-norm optimization problem. Therefore, the pulse shaping filters designed as (12) exhibit the good properties of minimum-norm estimator [17]. Moreover, its robustness in front of sensing errors is confirmed given the equivalence of (12) and total least-squares [18].

\section{IMPACT OF INCOMPLETE EXTERNAL-INTERFERENCE INFORMATION IN SIGNAL DETECTION}

In the previous Section, we have reviewed the uncoordinated design of pulse shaping filters in opportunistic networks. Because each internal user only considers local observations, the subspaces sensed at each node may differ. Hence, the noisesubspace bases sensed at each node can be written as

$$
\begin{aligned}
& \hat{\boldsymbol{\Psi}}_{\mathcal{N}, \mathrm{T}}=\left[\begin{array}{ll}
\boldsymbol{\Psi}_{\mathcal{N}}^{(0)} & \boldsymbol{\Delta}_{\mathrm{T}}
\end{array}\right], \\
& \hat{\boldsymbol{\Psi}}_{\mathcal{N}, \mathrm{R}}=\left[\begin{array}{ll}
\boldsymbol{\Psi}_{\mathcal{N}}^{(0)} & \boldsymbol{\Delta}_{\mathrm{R}}
\end{array}\right],
\end{aligned}
$$

where $\Psi_{\mathcal{N}}^{(0)}$ represents the DoF sensed as available at both internal nodes, and $\boldsymbol{\Delta}_{\mathrm{i}}$, for $\mathrm{i}=\{\mathrm{T}, \mathrm{R}\}$, contains those DoF that are sensed as available at only one internal node. Notice that $K_{\mathrm{i}}=K_{0}+\kappa_{\mathrm{i}}$, with $K_{0}=\operatorname{rank}\left(\boldsymbol{\Psi}_{\mathcal{N}}^{(0)}\right)$ and $\kappa_{\mathrm{i}}=\operatorname{rank}\left(\boldsymbol{\Delta}_{\mathrm{i}}\right)$. Recalling the design scheme reviewed in Sec. III, each internal user will have a set of $N$ possible pilot waveforms, namely pilot-book, such that

$$
\begin{aligned}
& \mathcal{P}_{\mathrm{T}}=\left\{\tilde{\boldsymbol{\varphi}}_{\mathrm{T}, 1}, \ldots, \tilde{\boldsymbol{\varphi}}_{\mathrm{T}, N}\right\} \in \hat{\mathcal{N}}_{\mathrm{T}}, \\
& \mathcal{P}_{\mathrm{R}}=\left\{\tilde{\boldsymbol{\varphi}}_{\mathrm{R}, 1}, \ldots, \tilde{\boldsymbol{\varphi}}_{\mathrm{R}, N}\right\} \in \hat{\mathcal{N}}_{\mathrm{R}},
\end{aligned}
$$

corresponding to the $N$ columns of orthogonal projectors onto $\hat{\mathcal{N}}_{\mathrm{T}}$ and $\hat{\mathcal{N}}_{\mathrm{R}}$, respectively. Note that $\hat{\mathcal{N}}_{\mathrm{T}} \neq \hat{\mathcal{N}}_{\mathrm{R}}$ whenever $\kappa_{\mathrm{i}} \neq 0$.

Therefore, when the receiving node uses their local pilots as reference signals, the detectability can be compromised. Moreover, receiver has to face the uncertainty exhibited by $\boldsymbol{\Delta}_{\mathrm{i}}$ to improve the performance of the opportunistic communication.

Regarding to the model presented in (2), the signal sensed by the receiver can be written as

$$
x(n)=\sum_{m} \sqrt{S_{\mathrm{R}}} a[m] \tilde{\varphi}_{\mathrm{T}, k}(n-m T)+v_{1}(n)+v_{2}(n),
$$

where $\tilde{\varphi}_{\mathrm{T}, k}(n)$ is the $k$-th element of $\mathcal{P}_{\mathrm{T}}, v_{1}(n)$ and $v_{2}(n)$ are the white complex noises from the common and uncommon DoF, respectively, assumed to be Gaussian random variables. It is worth noting that the receiver's performance can be improved by diminishing the noise enhancement introduced by $v_{2}(n)$.

Thus, the objectives of the internal system are twofold:

1) Since the transmitter will select an arbitrary pilot $\tilde{\varphi}_{\mathrm{T}, i}$ from $\mathcal{P}_{\mathrm{T}}$ with probability $\frac{1}{N}$, the receiver should find the pilot $\tilde{\varphi}_{\mathrm{R}, j}$ from $\mathcal{P}_{\mathrm{R}}$ such that

$$
\max _{j}\left|\tilde{\varphi}_{\mathrm{T}, i}^{H} \tilde{\varphi}_{\mathrm{R}, j}\right| \text {. }
$$

2) Recalling that $\hat{\mathcal{N}}_{\mathrm{T}} \neq \hat{\mathcal{N}}_{\mathrm{R}}$, the receiver should design a filter $\hat{\mathbf{w}}$, using the pilot selected in (16) as reference, such that only senses the $K_{0}$ DoF belonging to the intersection $\hat{\mathcal{N}}_{\mathrm{T}} \cap \hat{\mathcal{N}}_{\mathrm{R}}=\mathcal{N}_{0}$, i.e.

$$
\hat{\mathbf{w}} \in \mathcal{N}_{0} .
$$

Notice that this filter will (ideally) cancel noise sensed from uncommon dimensions. Furthermore, it can be used to assist the internal transmitting node to achieve subspace consensus via feedback.

Therefore, these two addressed objectives can be cast as a joint optimization problem, i.e.

$$
\left\{\hat{\mathbf{w}}_{\hat{\iota}}, \hat{\iota}\right\}=\underset{\left\{\mathbf{w}_{\iota}, \iota\right\}}{\arg \min } \mathbb{E}\left\{\left|\mathbf{w}_{\iota}^{H} \mathbf{x}(n)-d_{\mathrm{R}, \iota}(n)\right|^{2}\right\},
$$

for $\iota \in\{1, \ldots, N\}$. It is noteworthy that the reference signal at receiver $d_{\mathrm{R}, \iota}(n)$ is the $\iota$-th pilot from $\mathcal{P}_{\mathrm{R}}$, i.e. $\tilde{\varphi}_{\mathrm{R}, \iota}(n)$. Nevertheless, pilots from $\mathcal{P}_{\mathrm{R}}$ should exhibit certain invariances with respect to those from $\mathcal{P}_{\mathrm{T}}$ in order to be a valid reference signal, due to the lack of coordination between internal users.

\section{AnAlysis of Solution InVARIAnCE}

This Section is devoted to studying the invariance of the proposed pilot waveforms (12). As we have aforementioned, some invariances are required in order that (12) can be used as time-reference signals.

\section{A. Invariance to Rotations}

Because the proposed pilot waveforms rely on the orthogonal projector onto the noise subspace, they exhibit invariance to rotations when the sensed noise-subspace basis is acquired with a rotation within the noise subspace. This invariance is of paramount interest, because guarantees coherent detection.

The proof of this property can be found in [15], [16], and it is omitted in this conference presentation for space limitations. 


\section{B. Invariance to Subspace Uncertainties}

In the ideal scenario where $\kappa_{\mathrm{T}}=\kappa_{\mathrm{R}}=0$, the crosscorrelation between the received signal and the selected pilot from $\mathcal{P}_{\mathrm{R}}$ will be almost one, and solely degraded by the signalto-noise ratio (SNR). However, in the realistic scenario with sensing uncertainties, we will have a mismatching loss.

Hereunder, we analyze the penalty due to the lack of coordination between internal nodes.

Taking into account the noise-subspace bases decomposition in (13), the $\iota$-th element of $\mathcal{P}_{\mathrm{i}}$, for $\mathrm{i}=\{\mathrm{T}, \mathrm{R}\}$, is given by

$$
\tilde{\varphi}_{i, \iota}=\left(\mathbf{e}_{i, \iota}^{H}\left(\mathbf{P}_{\mathcal{N}}+\Upsilon_{i}\right) \mathbf{e}_{i, \iota}\right)^{-1 / 2}\left(\mathbf{P}_{\mathcal{N}}+\Upsilon_{i}\right) \mathbf{e}_{i, \iota}
$$

where $\mathbf{P}_{\mathcal{N}}$ is the orthogonal projector onto the intersection $\hat{\mathcal{N}}_{\mathrm{T}} \cap \hat{\mathcal{N}}_{\mathrm{R}}=\mathcal{N}_{0}$, and $\Upsilon_{\mathrm{i}}$ is the orthogonal projector onto the uncommon DoF subspace, namely $\overline{\mathcal{N}}^{\kappa_{\mathrm{i}}}$. Therefore, (19) admits the following decomposition

$$
\tilde{\varphi}_{\mathrm{i}, \iota}=\tilde{\varphi}_{\mathrm{i}, \iota}^{(0)}+\tilde{\varphi}_{\mathrm{i}, \iota}^{(\overline{\mathcal{N}})}
$$

where $\tilde{\varphi}_{\mathrm{i}, \iota}^{(0)}$ belongs to the intersection $\mathcal{N}_{0}$, and $\tilde{\varphi}_{\mathrm{i}, \iota}^{(\overline{\mathcal{N}})}$ to $\overline{\mathcal{N}}^{\kappa_{\mathrm{i}}}$.

Therefore, taking into account that

$$
\begin{aligned}
\overline{\mathcal{N}}^{\kappa_{\mathrm{T}}} \cap \overline{\mathcal{N}}^{\kappa_{\mathrm{R}}} & =\emptyset, \\
\overline{\mathcal{N}}^{\kappa_{\mathrm{i}}} \cap \mathcal{N}_{0} & =\emptyset, \text { for } \mathrm{i}=\{\mathrm{T}, \mathrm{R}\},
\end{aligned}
$$

the output of a matched-filter receiver can be written as

$$
\tilde{\boldsymbol{\varphi}}_{\mathrm{R}}^{H} \tilde{\boldsymbol{\varphi}}_{\mathrm{T}}=\frac{\mathbf{e}_{\mathrm{R}}^{H} \mathbf{P}_{\mathcal{N}} \mathbf{P}_{\mathcal{N}} \mathbf{e}_{\mathrm{T}}}{\sqrt{\left(\mathbf{e}_{\mathrm{R}}^{H} \mathbf{P}_{\mathcal{N}, \mathrm{R}} \mathbf{e}_{\mathrm{R}}\right)\left(\mathbf{e}_{\mathrm{T}}^{H} \mathbf{P}_{\mathcal{N}, \mathrm{T}} \mathbf{e}_{\mathrm{T}}\right)}} .
$$

According to the proposed noise-subspace bases decomposition in (13), a fraction $K_{0} / K_{\mathrm{i}}$ of the total pulse energy at the i-th internal user will be transmitted through the intersection $\mathcal{N}_{0}$, whereas the remaining fraction $\left(K_{\mathrm{i}}-K_{0}\right) / K_{\mathrm{i}}=\kappa_{\mathrm{i}} / K_{\mathrm{i}}$ will be lost. Finally, the matched-filter mismatch factor is given by

$$
\tilde{\boldsymbol{\varphi}}_{\mathrm{R}}^{H} \tilde{\boldsymbol{\varphi}}_{\mathrm{T}}=\left(1+\frac{\kappa_{\mathrm{T}}+\kappa_{\mathrm{R}}}{K_{0}}+\frac{\kappa_{\mathrm{T}} \kappa_{\mathrm{R}}}{K_{0}^{2}}\right)^{-1 / 2} .
$$

It is worth noting that, when $K_{0}$ is large enough, the secondorder term may be neglected. Therefore, due to those DoF belonging to the intersection are preserved (except a scaling factor), designed pilot waveforms are invariant to subspace uncertainties subject to an energy loss.

\section{SPARSITY-BASED JOINT CONSENSUS AND (Multi-)Signal Detection}

Traditionally, the cost function in (18) is solved by the wellknown mean-square error (MSE) optimal filter $\hat{\mathbf{w}}_{\iota}$, given the $\iota$-th reference signal. Therefore, the MSE in (18) given the Wiener solution of $\hat{\mathbf{w}}_{\iota}$ is given by

$$
\operatorname{MSE}\left(\iota \mid \hat{\mathbf{w}}_{\iota}\right)=P_{d}(\iota)-P_{y}(\iota),
$$

where $P_{d}(\iota)=\mathbb{E}\left\{\left|d_{\mathrm{R}, \iota}(n)\right|^{2}\right\}$ is the power of the $\iota$-th reference signal and $P_{y}(\iota)=\mathbb{E}\left\{\mathbf{p}(\iota)^{H} \mathbf{R}_{x x}^{-1} \mathbf{p}(\iota)\right\}$ is the power at the output of receiver's filter. Notice that $\mathbf{R}_{x x}$ is the input signal autocorrelation matrix, whereas $\mathbf{p}(\iota)=\mathbb{E}\left\{\mathbf{x}(n) d_{\mathrm{R}, \iota}^{*}(n)\right\}$ is the cross-correlation vector. Since $P_{d}(\iota)$ is independent of the input data, the signal detection problem is cast as

$$
\hat{\iota}=\underset{\iota \in\{1, \ldots, N\}}{\arg \max } \mathbb{E}\left\{\mathbf{p}(\iota)^{H} \mathbf{R}_{x x}^{-1} \mathbf{p}(\iota)\right\} .
$$

In other words, the receiver's pilot waveform which presents the highest correlation with the input signal has to be selected. Albeit it is out of the scope of this work, it is worth noting that this procedure can be accelerated by implementing a multipledwell scheme [19].

What it is very interesting to notice is that in our problem the waveform detection is an inherent sparse problem, specially when realistic (uncertainty-limited) scenarios are considered. Due to the partial knowledge of external-network state, the solution proposed in (25) is, in general, not optimal.

Note that, whenever $\kappa_{\mathrm{T}}, \kappa_{\mathrm{R}} \neq 0$, the SNR at receiver is given by

$$
\mathrm{SNR}_{\mathrm{R}}=\frac{\left(1-\frac{\kappa_{\mathrm{T}}}{K_{\mathrm{T}}}\right) S_{\mathrm{T}}}{\left(1+\frac{\kappa_{\mathrm{R}}}{K_{0}}\right) N_{0}}=\frac{1-\frac{\kappa_{\mathrm{T}}}{K_{\mathrm{T}}}}{1+\frac{\kappa_{\mathrm{R}}}{K_{0}}} \mathrm{SNR}_{0},
$$

where $\mathrm{SNR}_{0}$ is the nominal SNR (i.e. that when $\kappa_{\mathrm{T}}=\kappa_{\mathrm{R}}=0$ ), and $N_{0}$ is the noise power. Since the received signal can be written as

$$
\mathbf{x}_{m}=a[m] \sqrt{S_{\mathrm{T}}} \tilde{\boldsymbol{\varphi}}_{\mathrm{T}}+\boldsymbol{v}_{1}+\boldsymbol{v}_{2},
$$

and recalling the structure of pilot waveforms presented in (12), internal receiving node may estimate (27) as

$$
\hat{\mathbf{x}}=\tilde{\mathbf{P}}_{\mathcal{N}, \mathrm{R}} \boldsymbol{\alpha}
$$

where $\boldsymbol{\alpha}=\{0,1\}^{N}$ is a sparse vector that selects which pilot better estimates the input signal and $\tilde{\mathbf{P}}_{\mathcal{N}, \mathrm{R}}$ is a modified projector such that

$$
\tilde{\mathbf{P}}_{\mathcal{N}, \mathrm{R}}=\sum_{n=1}^{K_{\mathrm{R}}} \lambda_{n} \mathbf{P}_{n}
$$

with $\mathbf{P}_{n}=\left[\hat{\boldsymbol{\Psi}}_{\mathcal{N}, \mathrm{R}}\right]_{n}\left[\hat{\boldsymbol{\Psi}}_{\mathcal{N}, \mathrm{R}}\right]_{n}^{H}$ being the projector onto the $n$-th receiver's noise subspace singleton, where $\left[\hat{\boldsymbol{\Psi}}_{\mathcal{N}, \mathrm{R}}\right]_{n}$ is the $n$-th column of basis in (13b), and $\lambda_{n}$ is 0 or 1 depending on if the $n$-th singleton belongs to the intersection of noise subspaces or not. By defining $\boldsymbol{\lambda}=\left[\lambda_{1}, \ldots, \lambda_{K_{\mathrm{R}}}\right]^{T}$ and $\mathbf{P}=\left[\begin{array}{lll}\mathbf{P}_{1} & \cdots & \mathbf{P}_{K_{\mathrm{R}}}\end{array}\right]$, design problems in (28) and (29) can be jointly cast as

$$
\hat{\boldsymbol{\beta}}=\underset{\boldsymbol{\beta}}{\arg \min }\|\boldsymbol{\beta}\|_{1} \text { s.t. } \mathbf{x}=\mathbf{P} \boldsymbol{\beta},
$$

with $\boldsymbol{\beta}=\boldsymbol{\lambda} \otimes \boldsymbol{\alpha}$, being $\otimes$ the Kronecker tensor product. It is noteworthy that the complexity of the design problem have increased due to the stacking in (30). However, it is an inherent advantage since the receiver is now able to simultaneously detect up to $K$ orthogonal pilots from different users, which makes the discussed scheme feasible in multiuser scenarios.

As a last comment on (30), we note that it is sensitive to noise, specially in low-SNR regimes. Therefore, let us consider that receiver is able to capture $Q$ observations from the transmitter, and they are stacked in a $N Q$ column vector $\tilde{\mathbf{x}}=\left[\begin{array}{lll}\mathbf{x}_{1}^{T} & \cdots & \mathbf{x}_{Q}^{T}\end{array}\right]^{T}$. In addition, let us consider the extended matrix $\boldsymbol{\Phi}^{T}=\left[\begin{array}{lll}\mathbf{P} & \cdots & \mathbf{P}\end{array}\right]$, i.e. $\boldsymbol{\Phi}$ contains $Q$ times matrix $\mathbf{P}$ stacked in columns. Thus, the extended joint design problem becomes

$$
\hat{\boldsymbol{\beta}}=\underset{\boldsymbol{\beta}}{\arg \min }\|\boldsymbol{\beta}\|_{1} \text { s.t. }\|\tilde{\mathbf{x}}-\boldsymbol{\Phi} \boldsymbol{\beta}\|_{2}^{2} \leq \epsilon^{2} .
$$




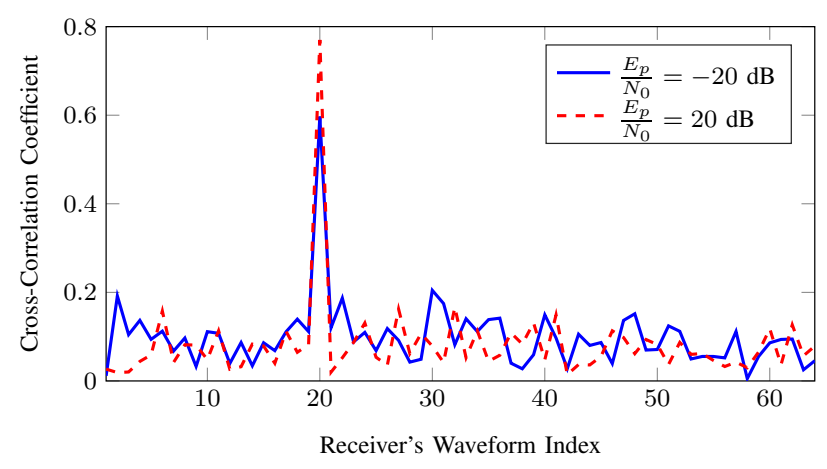

Fig. 2: Cross-correlation coefficient between received signal and all elements of the receiver's pilot-book in an uncertainty-free scenario, i.e. $\kappa_{\mathrm{T}}=\kappa_{\mathrm{R}}=0$.

It is worth noting that for large data block-length $Q$, the proposed extended optimization in (31) may burden computational inefficiency. Nevertheless, it is straightforward to see that the extended problem in (31) is equivalent to

$$
\hat{\boldsymbol{\beta}}=\underset{\boldsymbol{\beta}}{\arg \min }\|\boldsymbol{\beta}\|_{1} \text { s.t. } \sum_{q=1}^{Q}\left\|\mathbf{x}_{q}-\mathbf{P} \boldsymbol{\beta}\right\|_{2}^{2} \leq \epsilon^{2} .
$$

Therefore, the least-squares constraint of full $N Q$-length datablock in (31) can be tackled as the cumulative least-squares error of each $N$-length sub-block $\mathbf{x}_{q}$, yielding a reduced computational complexity with respect to (31).

\section{Simulation Results}

Numerical assessment of results derived in this work is presented in this Section.

\section{A. Matched-Filter Detection: Proof-of-concept}

The detection performance of the distributed pilot-based matched filter receiver is assessed herein. To do so, we consider an $M$-user heterogeneous external network transmitting in a 64-dimensional space. External-user DoF occupation is $3 / 8$, i.e. $D=24$ DoF. Therefore, the actual number of available $\mathrm{DoF}$ is $K=40$. For this proof-of-concept, two scenarios are considered: $E_{p} / N_{0}=-20 \mathrm{~dB}$ and $E_{p} / N_{0}=20 \mathrm{~dB}$, with $E_{p} / N_{0}$ being the pulse energy to noise ratio. Furthermore, we assume that internal transmitting node has arbitrarily selected the 20-th column of its noise-subspace projector.

In Fig. 2 we depict the cross-correlation coefficient between the received signal and the $N$ elements of the receiver's pilotbook $\mathcal{P}_{\mathrm{R}}$ in an ideal scenario, i.e. $\kappa_{\mathrm{T}}=\kappa_{\mathrm{R}}=0$. As it can be appreciated, the correct detection is guaranteed.

Concerning more realistic scenarios, we illustrate the crosscorrelation coefficient under DoF sensing uncertainties in Fig. 3. Regarding to the uncertainty, let $\rho_{\mathrm{i}}$ be the relative uncertainty at the $\mathrm{i}$-th internal node, defined as $\rho_{\mathrm{i}} \triangleq \frac{\kappa_{\mathrm{i}}}{K \mathrm{i}}$, for $\mathrm{i}=\{\mathrm{T}, \mathrm{R}\}$.

For this particular example, we have considered $\rho_{\mathrm{T}}=$ $5 / 45=11.11 \%$ and $\rho_{\mathrm{R}}=15 / 55=27.27 \%$. It is worth noting that $\rho_{\mathrm{T}}$ degrades the transmitted $E_{p}$, whereas $\rho_{\mathrm{R}}$ is a source of noise enhancement. As we may observe, although the detector exhibits a good performance in high- $\frac{E_{p}}{N_{0}}$ regimes, the detection can be compromised in low- $\frac{E_{p}}{N_{0}}$ regimes.

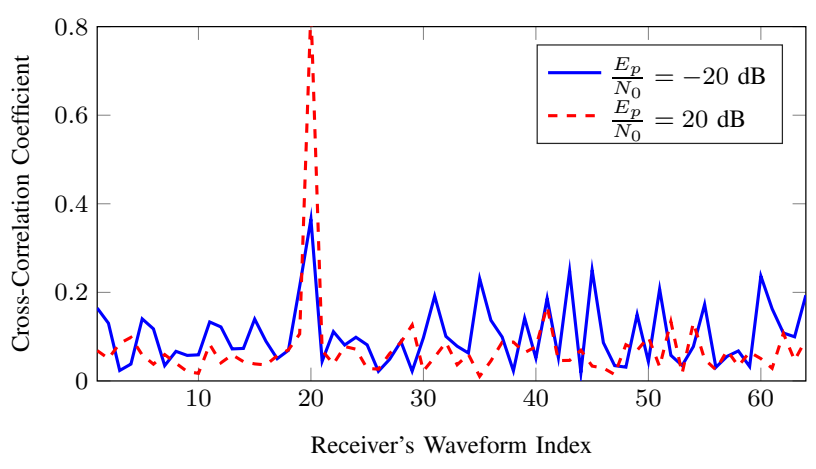

Fig. 3: Cross-correlation coefficient between received signal and all elements of the receiver's pilot-book with uncertainty $\left(\kappa_{\mathrm{T}}, \kappa_{\mathrm{R}} \neq 0\right)$.

\section{B. Uncertainty Analysis}

As we appreciate in the previous Subsection, DoF sensing uncertainty degrades the detection performance, as we have analytically derived in Sec. V, eq. (23). Herein, we numerically assess the impact of the performance loss on the received signal-to-noise ratio as a function of the absolute uncertainty at receiver and for different absolute uncertainties at transmitter. Let us consider the scenario presented in Sec. VII-A. In Fig. 4 we compare the theoretical loss and a Montecarlo simulation of 100,000 independent realizations. It is worth noting that, for high noise level from uncommon DoF, the mismatch severely degrades the system performance, specially in low- $\frac{E_{p}}{N_{0}}$ regimes.

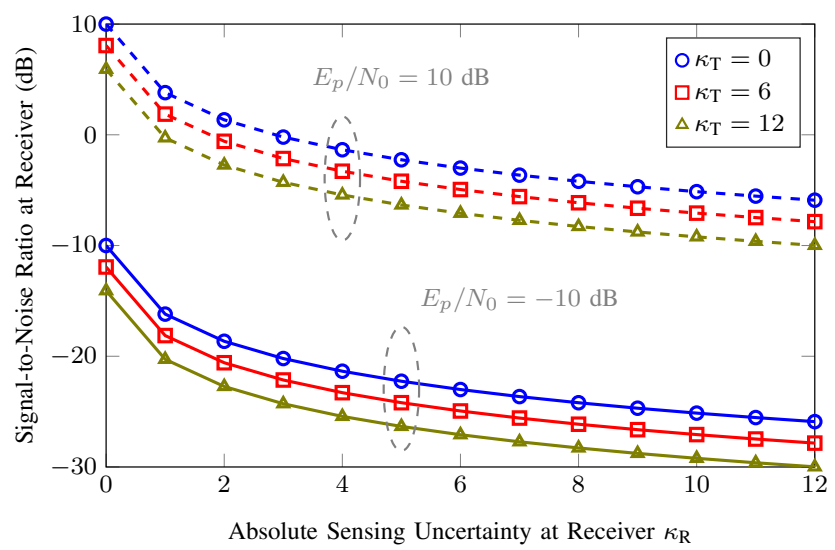

Fig. 4: Signal-to-Noise Ratio at Receiver in a realistic scenario. Lines and markers represent theoretical and simulated values, respectively.

\section{Degrees-of-Freedom Detection Performance}

In order to combat sensing uncertainties, we have proposed a joint $\ell_{1}$-norm optimization (32). This procedure is able to jointly detect which DoF are simultaneously used by transmitter and receiver and select the pulse shape that better detects the received signal. We assume the same scenario as in Sec. VII-A, i.e. $N=64 \mathrm{DoF}$ and external-network occupation of $3 / 8$. For simplicity, we have assumed $\rho_{\mathrm{T}}=\rho_{\mathrm{R}}=12 / 52$.

Herein, we assess the capacity of the proposed algorithm to differentiate which DoF are simultaneously used by both transmitter and receiver. To do so, let us define the probability of correct detection $P_{\mathrm{D}}$ as the probability of detecting as active 


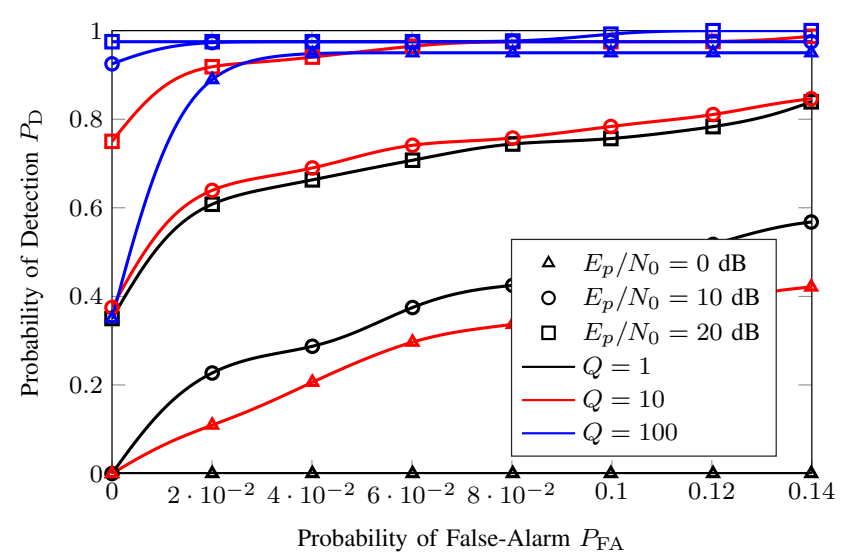

Fig. 5: ROC for $E_{p} / N_{0}=\{0,10,20\} \mathrm{dB}$ and different block-length $Q=\{1,10,100\}$ averaged over $10^{4}$ independent realizations.

a DoF belonging to the intersection. Likewise, we refer to the miss-detection probability $P_{\mathrm{MD}}$ as the probability of not detecting as active a DoF belonging to the intersection.

In Fig. 5 we have depicted the Receiver Operating Characteristics (ROC) of the proposed $\ell_{1}$-norm algorithm (32). In these simulations, we have considered different $E_{p} / N_{0}$ and different block-lengths $Q$. The probability of false-alarm $P_{\mathrm{FA}}$ is set as the probability of detecting as active a DoF not belonging to the intersection. In order to maximize $P_{\mathrm{D}}$, we have considered a Neyman-Pearson threshold, i.e.

$$
\gamma=\sqrt{\left(\sigma_{\mathrm{R}}^{2} / Q\right)} \mathcal{Q}^{-1}\left(P_{\mathrm{FA}}\right),
$$

with $\mathcal{Q}(\cdot)$ being the tail probability of a Gaussian distribution. Notice that $\sigma_{\mathrm{R}}^{2}$ is the noise variance at receiver. The missdetection probability $P_{\mathrm{MD}}$, defined as the probability of detecting a DoF not belonging to the intersection is depicted in Fig. 6 as a function of $E_{p} / N_{0}$. For this particular example, we have considered $P_{\mathrm{FA}}=\{0.1,0.001\}$, i.e. $\gamma^{\prime}=\mathcal{Q}^{-1}\left(P_{\mathrm{FA}}\right)=$ $\{0.84,2.88\}$. It is worth noting that, even for small $P_{\mathrm{FA}}, P_{\mathrm{MD}}$ rapidly diminishes with $E_{p} / N_{0}$.

\section{CONCLUSIONS}

This paper has addressed the distributed scenario-adapted design of pilot reference signals in opportunistic communications. The use of these pilots as reference signals relies on the existence of certain invariances. Furthermore, although matched filter is optimal in white noise, sensing uncertainties worsen the performance by means of energy loss and noise enhancement. The latter can be solved by jointly selecting which DoF have two users in common and detecting the presence of such user. As we have seen, this extended $\ell_{1}$-norm problem enables the proposed technique in multiuser scenarios.

\section{REFERENCES}

[1] L. Lu, G. Y. Li, A. Maaref, and R. Yao, "Opportunistic transmission exploiting frequency- and spatial-domain degrees of freedom," IEEE Wireless Commun., vol. 21, no. 2, pp. 91-97, April 2014.

[2] Y. Zhang, E. DallAnese, and G. B. Giannakis, "Distributed optimal beamformers for cognitive radios robust to channel uncertainties," IEEE Trans. Signal Process., vol. 60, no. 12, pp. 6495-6508, Dec 2012.

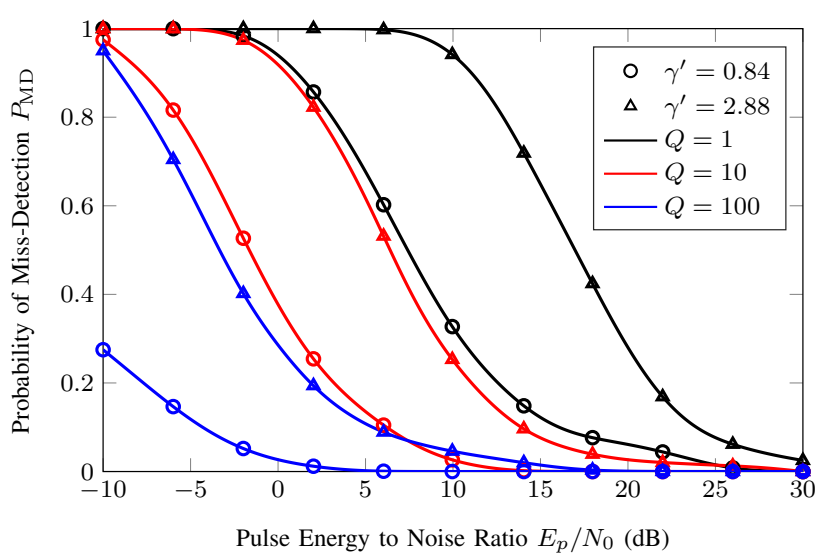

Fig. 6: Probability of Miss-Detection versus $E_{p} / N_{0}$ for $\gamma^{\prime}=\{0.84,2.88\}$ and block-length $Q=\{1,10,100\}$ averaged over $10^{4}$ independent realizations.

[3] L. S. Cardoso, M. Kobayashi, F. R. P. Cavalcanti, and M. Debbah, "Vandermonde-subspace frequency division multiplexing for two-tiered cognitive radio networks," IEEE Trans. Commun., vol. 61, no. 6, pp. 2212-2220, June 2013.

[4] R. Yao, Y. Liu, L. Lu, G. Y. Li, and A. Maaref, "Cooperative precoding for cognitive transmission in two-tier networks," IEEE Trans. Commun., vol. 64, no. 4, pp. 1423-1436, April 2016.

[5] M. Medard, "The effect upon channel capacity in wireless communications of perfect and imperfect knowledge of the channel," IEEE Trans. Inf. Theory, vol. 46, no. 3, pp. 933-946, May 2000.

[6] I. Hwang, B. Song, and S. S. Soliman, "A holistic view on hyper-dense heterogeneous and small cell networks," IEEE Commun. Mag., vol. 51, no. 6, pp. 20-27, June 2013.

[7] N. Yang, L. Wang, G. Geraci, M. Elkashlan, J. Yuan, and M. D. Renzo, "Safeguarding 5G wireless communication networks using physical layer security," IEEE Commun. Mag., vol. 53, no. 4, pp. 20-27, April 2015.

[8] O. Simeone, U. Spagnolini, Y. Bar-Ness, and S. H. Strogatz, "Distributed synchronization in wireless networks," IEEE Signal Process. Mag., vol. 25, no. 5, pp. 81-97, September 2008.

[9] J. Jose, A. Ashikhmin, T. L. Marzetta, and S. Vishwanath, "Pilot contamination and precoding in multi-cell TDD systems," IEEE Trans. Wireless Commun., vol. 10, no. 8, pp. 2640-2651, August 2011.

[10] X. Guo, S. Chen, J. Zhang, X. Mu, and L. Hanzo, "Optimal pilot design for pilot contamination elimination/reduction in large-scale multipleantenna aided OFDM systems," IEEE Trans. Wireless Commun., vol. 15, no. 11, pp. 7229-7243, Nov 2016.

[11] S. Haghighatshoar and G. Caire, "Massive MIMO pilot decontamination and channel interpolation via wideband sparse channel estimation," IEEE Trans. Wireless Commun., vol. 16, no. 12, pp. 8316-8332, Dec 2017.

[12] L. S. Muppirisetty, T. Charalambous, J. Karout, G. Fodor, and $\mathrm{H}$. Wymeersch, "Location-aided pilot contamination avoidance for massive MIMO systems," IEEE Trans. Wireless Commun., vol. 17, no. 4, pp. 2662-2674, April 2018.

[13] R. G. Gallager, Information Theory and Reliable Communications. John Wiley \& Sons, 1968.

[14] D. Tse and P. Viswanath, Fundamentals of Wireless Communication. Cambridge University Press, 2005.

[15] J. Borràs, J. Font-Segura, J. Riba, and G. Vázquez, "Dimension spreading for coherent opportunistic communications," in 201751 st Asilomar Conf. Signals, Syst. Comput., Oct 2017, pp. 1940-1944.

[16] J. Borràs and G. Vázquez, "Uncoordinated space-frequency pilot design for multi-antenna wideband opportunistic communications," in 2018 IEEE 19th Int. Workshop Signal Process. Adv. Wireless Commun. (SPAWC), Jun 2018, pp. 1-5.

[17] D. W. Tufts and R. Kumaresan, "Estimation of frequencies of multiple sinusoids: making linear prediction perform like maximum likelihood," Proc. IEEE, vol. 70, no. 9, pp. 975-989, Sept 1982.

[18] E. M. Dowling and R. D. DeGroat, "The equivalence of the total least squares and minimum norm methods," IEEE Trans. Signal Process., vol. 39, no. 8, pp. 1891-1892, Aug 1991.

[19] A. J. Viterbi, CDMA: principles of spread spectrum communication. Addison-Wesley Reading, MA, 1995, vol. 122. 